\title{
A speckle-imaging search for close triple companions of cataclysmic binaries
}

\author{
Michael M. Shara ${ }^{\circledR},{ }^{1 \star}$ Steve B. Howell, ${ }^{2}$ Elise Furlan, ${ }^{3}$ Crystal L. Gnilka, ${ }^{2}$ Anthony F. J. Moffat, ${ }^{4}$ \\ Nicholas J. Scott ${ }^{2}$ and David Zurek ${ }^{1}$ \\ ${ }^{1}$ Department of Astrophysics, American Museum of Natural History, Central Park West at 79th Street, New York, NY 10024, USA \\ ${ }^{2}$ NASA Ames Research Center, Moffett Field, CA 94035, USA \\ ${ }^{3}$ NASA Exoplanet Science Institute, Caltech/IPAC, Mail Code 100-22, 1200 E. California Blvd., Pasadena, CA 91125, USA \\ ${ }^{4}$ Département de Physique et Centre de Recherche en Astrophysique du Québec, Université de Montréal, Montréal, QC H3C 3J7, Canada
}

Accepted 2021 July 28. Received 2021 July 28; in original form 2021 May 7

\begin{abstract}
The orbital periods of most eclipsing cataclysmic binaries are not undergoing linear secular decreases of order a few parts per billion as expected from simple theory. Instead, they show several parts per million increases and decreases on time-scales of years to decades, ascribed to magnetic effects in their donors, triple companions, or both. To directly test the triple companion hypothesis, we carried out a speckle-imaging survey of six of the nearest and brightest cataclysmic variables. We found no mainsequence companions earlier than spectral types M4V in the separation range $\sim 0.02-1.2 \operatorname{arcsec}$, corresponding to projected linear separations of $\sim 2-100 \mathrm{au}$, and periods of $\sim 3-1000 \mathrm{yr}$. We conclude that main-sequence triple companions to cataclysmic variables are not very common, but cannot rule out the presence of the faintest $\mathrm{M}$ dwarfs or close brown dwarf companions.
\end{abstract}

Key words: techniques: high angular resolution-binaries: close-stars: dwarf novae-novae, cataclysmic variables.

\section{INTRODUCTION}

Almost all cataclysmic variables (CVs) comprised red dwarfs (RD) or red giants transferring hydrogen-rich matter on to the surfaces of their white dwarf (WD) companions (Warner 1995). Mass transfer in these binaries (Walker 1954; Kraft 1962, 1964) drives a rich variety of phenomena. The most spectacular of these include the reasonably well understood accretion-powered outbursts of dwarf novae (Osaki 1974; Dubus, Otulakowska-Hypka \& Lasota 2018) and the thermonuclear-powered eruptions of classical novae (Starrfield et al. 1972; Prialnik, Shara \& Shaviv 1978).

Until the advent of large CCDs and large-area synoptic studies, many CVs (other than novae) were found because of their strong emission lines (Stephenson \& Sanduleak 1977) or very blue colours (Green, Schmidt \& Liebert 1986). Most CVs are now detected via their outbursts in wide-area synoptic surveys (Drake et al. 2014; Mróz et al. 2015a, b).

Five to ten erupting Galactic classical novae are usually detected in any given year... often by amateur astronomers. A germinal wide area, infrared survey of the Milky Way has recently shown that the Galactic nova eruption rate is $46 \pm 12$ novae $\mathrm{yr}^{-1}$ (De et al. 2021). This confirms that $\sim 80-90$ percent of Galactic novae have been missed over the past century because they are distant and reddened. Deep, high cadence all-sky visible-light surveys, and infrared surveys will greatly increase the outbursting Galactic nova and dwarf nova detection rates in the coming decade.

^E-mail: mshara@amnh.org
GAIA confirms that the closest known of all CVs (WZ Sge) is $43 \mathrm{pc}$ distant, consistent with a space density of CVs that is $\sim 5 \times 10^{-5} \mathrm{pc}^{-3}$ (Pala et al. 2020), unless most CVs 'hibernate' as detached RD-white binaries during most of the time between successive nova eruptions (Shara et al. 1986; Hillman, Shara, Prialnik \& Kovetz 2020). This GAIA-based space density is $\sim$ four orders of magnitude lower than that of main-sequence stars near the Sun. Thus, in zeroth approximation, 1 star in 10000 near the Sun is currently a mass-transferring CV.

\subsection{CV orbital periods}

Other phenomena associated with CVs are much less wellunderstood. As expected for a collection of binaries with randomly oriented orbital planes, $\sim 10-15$ percent of all CVs are deeply eclipsing. Individual eclipsing systems' orbital periods $P$ can be measured, over a baseline of $\sim$ a decade, with precisions of 1 part in $10^{7}$. The orbital period distributions of all subtypes of CVs, and their various subclasses, are important tests of evolutionary models of CVs. Population synthesis and binary-evolution codes can be coupled with angular momentum loss prescriptions (Schreiber, Zorotovic \& Wijnen 2016) to produce predictions of the orbital period distributions of CVs (Howell, Nelson \& Rappaport 2001; Goliasch \& Nelson 2015; Schreiber et al. 2016). The models' predictions are poor matches to the observed orbital period distributions of all CVs (Schreiber et al. 2016), dwarf novae (Knigge, Baraffe \& Patterson 2011), and classical novae (Fuentes-Morales et al. 2021). In particular, the ratio of short to long-period CVs, the observed strong peak of novae in the 3-4 h period range (Fuentes-Morales et al. 2021) and the shortage of dwarf novae in the $2-4 \mathrm{~h}$ period 
range (Knigge et al. 2011) are seriously discrepant with models' predictions.

\subsection{CVs' $\mathrm{d} P / \mathrm{d} t$}

The rates of change of $\mathrm{CV}$ orbital periods $\mathrm{d} P / \mathrm{d} t$ are also not well-understood. CVs' $P$ are predicted to be secularly decreasing because the underlying binaries are shedding angular momentum (Howell et al. 2001; Knigge et al. 2011) via their secondaries' winds (Verbunt \& Zwaan 1981) and gravitational radiation (Paczynski \& Sienkiewicz 1981). CVs born with $P \sim 10$ h should reach $P \sim 3 \mathrm{~h}$ in $\sim 1$ Gyr (Knigge et al. 2011; Hillman, Shara, Prialnik \& Kovetz 2020). This corresponds to $\mathrm{d} P / \mathrm{d} t$ of order $-10^{-11}$ cycles $\mathrm{d}^{-1}$, or a few parts per billion (ppb) $\mathrm{yr}^{-1}$. Contrary to expectations, CVs $P$ that have been monitored for decades show orbital period decreases, increases, and cyclical variations. Borges et al. (2008) lists 14 wellknown eclipsing CVs which display orbital period modulations with amplitudes as large as 7.9 parts per million (ppm) on time-scales of 5-35 yr. Updates and new additions to this list are given by Pilarčík et al. (2012), Boyd (2012), Bruch (2014), Pilarčík et al. (2018), Patterson et al. (2018), and (Schaefer 2020). Borges et al. (2008) adds '...there (are) presently no CVs with well sampled and precise $(\mathrm{O}-\mathrm{C})$ diagrams covering more than a decade of observations that do not show cyclical period changes'. The amplitudes and oscillatory frequencies of the $\mathrm{d} P / \mathrm{d} t$ of all classes of $\mathrm{CVs}$ show no clearcut patterns.

\subsection{Explaining $\mathrm{d} P / \mathrm{d} t$}

\subsubsection{Starspots}

One possible explanation for the modulations of CVs' periods is magnetic activity cycles and star-spots in their hydrogen-rich donors (Applegate 1992; Livio \& Pringle 1994). These can greatly vary mass and angular momentum transfer rates on time-scales of years. Tomographic studies provide support for this hypothesis, showing large star-spots on the secondaries of BV Cen, V426 Oph, and SS Cyg (Watson et al. 2007a, b; Hill 2016).

\subsubsection{Triple companions}

A second (and non-exclusionary) explanation is that CVs may display positive or oscillatory $\mathrm{d} P / \mathrm{d} t$ because they are in triple-star systems. The distant companion of a CV with years-to-centuries long orbits would periodically displace the center of mass of the $\mathrm{CV}$ to approach and then recede from Earth. The variable light travel time to a CV in a triple or multiple-star system would yield 'early' and 'late' eclipses, and variations in $\mathrm{d} P / \mathrm{d} t$. Triple companions at linear separations from CVs' centers of mass of 3,10, and 30 au would experience orbital periods of $\sim 5,30$, and $160 \mathrm{yr}$, respectively.

Triple companions are not as far-fetched as they might seem at first glance - there are 3 triple-star systems (and 7 binaries) amongst the 21 star systems closest to Earth (Henry et al. 2018). Even higher order multiples are remarkably common (Tokovinin 2018). Five of the nine nearest triple or higher multiple-star systems (GJ 570C, GJ 663, GJ 644, G 041-014, and LP 771-095) contain tertiary components certain or possibly of spectral type M4V or earlier, ${ }^{1}$ corresponding to our observational detection limits (see below).

\footnotetext{
${ }^{1}$ http://www.astro.gsu.edu/RECONS/TOP100.posted.htm
}

Most current WDs in CVs must have been red giants with radii $\geq 1-$ 3 au which engulfed their companions in the past (Meyer \& MeyerHofmeister 1979; Paczynski \& Sienkiewicz 1981; Law \& Ritter 1983). Current triple companions to CVs that were sufficiently distant from the common envelope binary ( $\geq 3$ au from the red giant) to have avoided engulfment then would have spiraled out to distances $\sim 2-$ 3 times larger due to mass loss from the inner, pre-CV binary. Closer in triple companions could have stayed at $\sim$ the same separation, or even moved closer in if they, too, were engulfed by the expanding atmosphere of the red giant. Theoretical considerations (Leigh et al. 2020) and numerical simulations of the complex evolution of triple stars (Hamers et al. 2021) make it clear that post common envelope binaries (PCEB) and CVs' triple companions of all masses (less than the initially most massive star in the triple system) and separations are possible.

\subsubsection{CV, PCEB, and EL CVn triples}

Examples of CVs suggested to be triple on the basis of variable $\mathrm{d} P / \mathrm{d} t$ include CH Cyg (Skopal et al. 1998), VY Scl (Martínez-Pais et al. 2000), V542 Cyg (Thorstensen, Peters \& Skinner 2010), FS Aur (Chavez et al. 2012, 2020), V2051 Oph (Qian et al. 2015), U Gem (Warner 1988; Dai \& Qian 2009), and EM Cyg (Liu et al. 2021). The masses of companions required to produce the observed $\mathrm{d} P / \mathrm{d} t$ range from those of giant planets, through brown dwarfs, up to $\sim 0.8 \mathrm{M}_{\odot}$.

Eclipsing PCEB that will become CVs also display large departures from expected mid-eclipse times. (Zorotovic \& Schreiber 2013) provided a comprehensive overview of eclipse timing variations in PCEB. They found that a remarkable $\sim 90$ per cent of PCEBs exhibit period variations. If these variations are due to tertiary companions, their masses are likely in the planetary or brown dwarf mass range. The best-studied example to date is NN Ser (Parsons et al. 2010). Beuermann et al. (2010) found excellent agreement with the observed period changes of NN Ser via two additional bodies with masses several times that of Jupiter superposed on the linear ephemeris of the binary. Remarkably, Marsh et al. (2014) found that the two-planet model correctly predicted a progressive lag in eclipse times of $36 \mathrm{~s}$ that set in since 2010 compared to the previous $8 \mathrm{yr}$ of precise times.

Two PCEB binaries that are particularly instructive are Wolf 1130 and V471 Tau. On the one hand, there is no doubt of the triple nature of the pre-CV Wolf 1130 (Mace et al. 2018), located just $16.6 \mathrm{pc}$ distant from Earth. Wolf 1130 comprised a T8 brown dwarf 3000 au from, and in orbit around an M subdwarf - ONe WD, which is itself in a 0.4967 day orbit. In contrast, the nearby PCEB, pre-CV binary V471 Tau has also often been cited as a triple-star candidate (Kundra \& Hric 2011). However, recently very sensitive observations have ruled out even a brown dwarf companion (Hardy et al. 2015) with a high degree of confidence.

The EL CVn eclipsing binaries, which are closely related to PCEBs, contain an A- or F-type star and a very low mass $\left(\sim 0.2 \mathrm{M}_{\odot}\right)$ pre-Helium WD. The low pre-WD masses mean that EL CVn stars must form from dynamically stable-mass transfer when the more massive star of the initial main-sequence binary was at the end of its main-sequence lifetime, or had just entered the sub giant branch (Chen et al. 2017), with the orbital period of the progenitor binary $<3$ days. Virtually all close main-sequence stars with orbital periods $<3$ days are known to be the inner binaries of hierarchical triple systems (Tokovinin et al. 2006). Lagos et al. (2020) examined five EL $\mathrm{CVns}$ in detail and found that all had nearby companions, consistent with K-M dwarfs. 
Table 1. Gemini speckle observations of six CVs.

\begin{tabular}{lcccccccc}
\hline Object & CV type & $V_{\text {min }}(\mathrm{mag})$ & Period $(\mathrm{min})$ & \multicolumn{1}{c}{ Dist. $(\mathrm{pc})$} & Date observed & Obs. time $(\mathrm{min})$ & $\lambda_{\text {filter }}$ & $\Delta$ mag @ 1 arcsec \\
\hline IX Vel & NL & 10.1 & 279.25 & $90.6 \pm 0.2$ & $2020-$ Nov-29 & 30 & $653 / 832$ & $6.5 / 8.2$ \\
AE Aqr & IP & 10.4 & 592.78 & $91.2 \pm 0.5$ & 2020-Jun-13 & 20 & $466 / 716$ & $5.0 / 8.4$ \\
U Gem & UG & 8.5 & 254.74 & $93.4 \pm 0.3$ & $2020-$-Feb-17 & 35 & $466 / 716$ & $4.5 / 5.9$ \\
SS Cyg & UG & 8.0 & 396.19 & $114.6 \pm 0.6$ & 2020-Jun-15 & 30 & $466 / 716$ & $4.8 / 7.8$ \\
V884 Her & AM & 12.8 & 113.01 & $115.1 \pm 0.3$ & $2020-J u n-11$ & 8 & $466 / 716$ & $4.9 / 5.1$ \\
AH Her & UGZ & 10.8 & 371.69 & $324.3 \pm 3.3$ & 2020-Feb-18 & 22 & $466 / 716$ & $3.7 / 5.7$ \\
\hline
\end{tabular}

Notes. Column 2: $\mathrm{NL}=$ novalike variable; $\mathrm{IP}=$ intermediate polar; $\mathrm{UG}=\mathrm{U}$ Geminorum-type dwarf nova;

$\mathrm{AM}=\mathrm{AM}$ Herculis $\mathrm{CV}=$ polar; $\mathrm{UGZ}=\mathrm{Z}$ Camelopardalis-type dwarf nova.

Column 3: CV's brightest $\mathrm{V}$ magnitude.

Column 8: Filter central wavelength (nm).

\subsection{Motivation and outline of this study}

Claims of triple companions to most CVs and PCEBs will require decades to centuries to resolve if the only evidence remains eclipse timings. The recent availability of new, very high resolution imaging facilities capable of detecting companions 5 (10) mag fainter than CV binaries at separations as small as 17 (1000) mas prompted us to search for such companions amongst the closest and brightest known CVs. These angular separations correspond to linear separations of putative triple companions that are relevant for $\mathrm{CV} P$ changes on interesting time-scales (see Section 3).

In Section 2, we briefly describe the speckle cameras, targets, and observations of this study. In Section 3, we show the results of our observations, placing stringent limits on optical companions to six of the brightest and closest known CVs. We briefly summarize our results in Section 4.

\section{TARGETS AND OBSERVATIONS}

In this first exploratory study, we observed six of the closest and best-studied CVs. They and their CV subtypes are IX Velorum (the closest novalike variable), AE Aquarii (an Intermediate Polar in a 'propeller' binary), U Geminorum (the prototypical U Gem-type dwarf nova, and suggested to have a triple companion (Dai \& Qian 2009)), SS Cygni (the brightest dwarf nova), V884 Her (one of the closest highly magnetic-WD Polars), and AH Herculis (one of the closest Z Cam-type CVs). In Table 1, we list these six targets, their $\mathrm{CV}$ subtypes, orbital periods, GAIA-determined distances, the dates of observation, integration time, the central wavelengths of the filters used to observe each target, and lower limits in the blue and red passbands on how much fainter any undetected companions must be at 1.2 arcsec from the CVs.

The observations were carried out with the twin speckle cameras Zorro and 'Alopeke located at the twin 8-m Gemini-S and Gemini$\mathrm{N}$ telescopes, at Cerro Pachon, Chile, and Mauna Kea, Hawaii, respectively. 'Alopeke and Zorro are dual-channel imagers using two electron-multiplying CCDs as the detectors. Each provides simultaneous two-colour, diffraction-limited optical imaging (FWHM $\sim 0.017 \mathrm{arcsec}$ at $550 \mathrm{~nm}$ ) of targets as faint as $V \sim 17$ over a 6.7 arcsec field of view. Each camera can also resolve wider companions (0.2$1.5 \mathrm{arcsec}$ ) with very large magnitude differences (up to 8-10 mag). Each has filter wheels providing bandpass limited observations. See Scott et al. (2018) for a detailed description of the instruments and their filters, as well as the 'Alopeke-Zorro Web pages. ${ }^{2}$ Each observation comprised multiple sets of 1000 exposures of $60 \mathrm{~ms}$ each, as well as calibration observations. The data reductions are

\footnotetext{
${ }^{2} \mathrm{https} / / / w w w . g e m i n i . e d u / i n s t r u m e n t a t i o n / a l o p e k e-z o r r o$
}

described in Horch et al. (2012) and the final data products are discussed in Howell et al. (2011).

\section{RESULTS}

The results of all observations are shown in Fig. 1. The red and blue curves in each figure correspond to the two filters used in each observation. They measure the contrast limits $\Delta \mathrm{m}$ achieved in magnitudes below the primary target. The reconstructed images, covering a $\sim 1.5 \operatorname{arcsec} \times 1.5$ arcsec region centered on each $\mathrm{CV}$ are also shown. In several cases (most notably AE Aqr and IX Vel), the surrounding background appears asymmetric and/or 'ringlike'. These 'features' are at the noise limit of the images, and are not real.

The key result of this paper is that no close, main-sequence triple companions (earlier than spectral type M4V) to any of the six observed target CVs were detected in the separation range 0.02-1.2 arcsec. Our observations are more sensitive in the redder filter, where very close companions $(\rho<0.1 \mathrm{arcsec})$ within 3-5 mag of the CVs are excluded. Farther out $(0.1 \operatorname{arcsec}<\rho<1.2$ arcsec), companions 5-8 mag fainter than the CVs would have been detected.

Five of the six observed CVs (IX Vel, AE Aqr, SS Cyg, U Gem, and V884 Her) are all located at about the same distance from Earth: $100+10$ pc. At $100 \mathrm{pc}$ the angular separations $0.02,0.1$, and 0.3 arcsec correspond to projected linear separations of 2,10 , and $30 \mathrm{au}$, respectively. Triple companions at those distances would experience orbital periods $\sim 3,30$, and $160 \mathrm{yr}$, respectively, similar to those noted for putative $\mathrm{CV}$ triple companions in the introduction.

We conclude by noting that our observations are not sensitive to brown dwarfs or the faintest $\mathrm{M}$ dwarfs; high- resolution infrared techniques will be needed to search for such companions to CVs.

\section{SUMMARY AND CONCLUSIONS}

Tertiary companions from planets to brown dwarfs to RD are known or suspected to exist around close binaries. If present, they cause orbital period variations of the inner binary. Such variations are observed for many CVs, though they might be due to other causes. To test whether close stellar companions to CVs might be common, we carried out visible-light speckle observations of six of the nearest/brightest known CVs to try to image nearby triple companions.

No companions were found in the angular separation range $\sim 0.02$ $1.2 \mathrm{arcsec}$, corresponding to physical separations of $\sim 2-100 \mathrm{au}$, and orbital periods of triple companions of $\sim 3-1000 \mathrm{yr}$. While far from definitive, this small survey demonstrates that main-sequence triple companions to $\mathrm{CVs}$, of spectral types earlier than $\mathrm{M} 4 \mathrm{~V}$, are not common. Our survey is not sensitive to the faintest $\mathrm{M}$ dwarfs or brown dwarfs, where high-resolution, near-infrared techniques must be employed. 

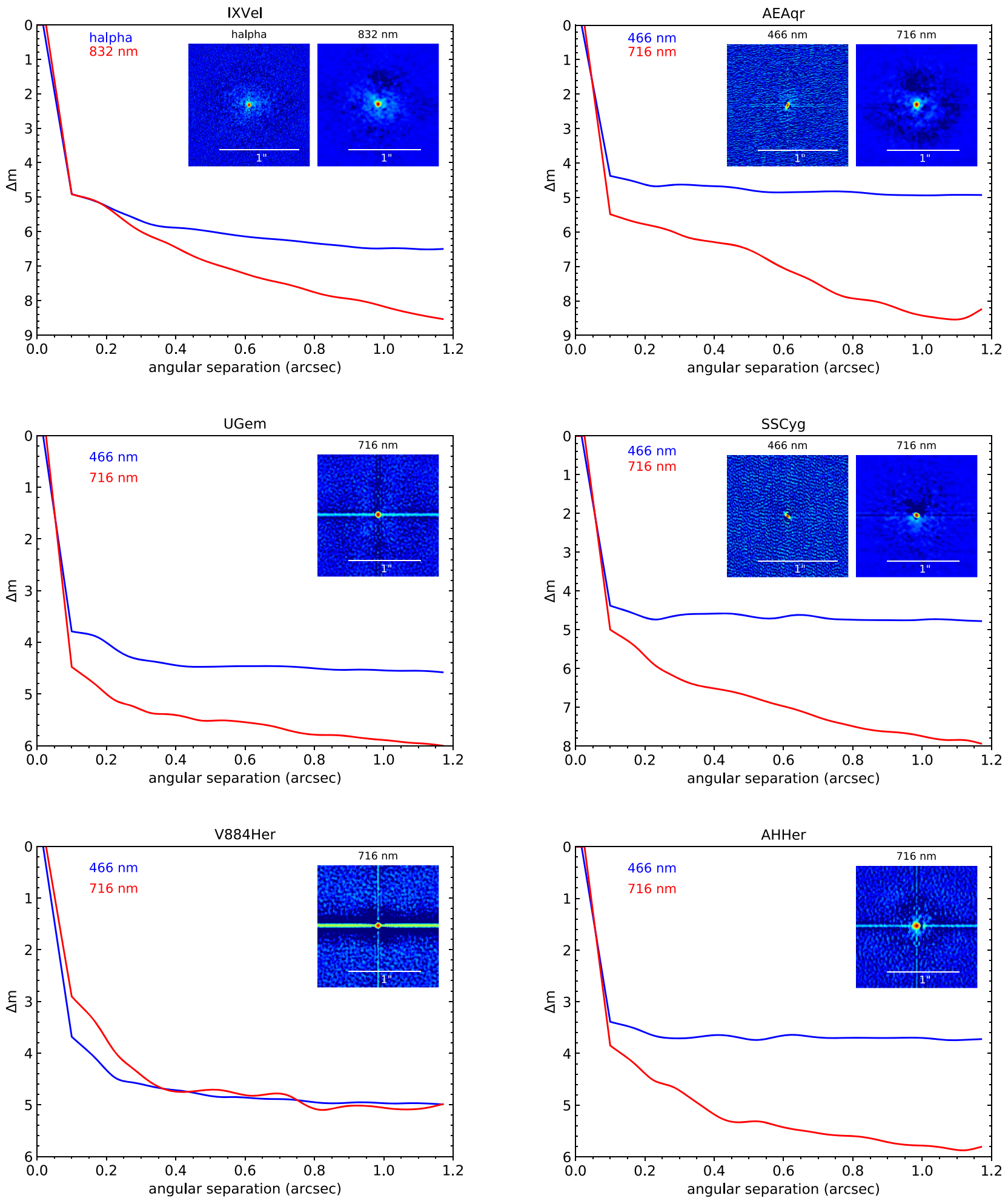

Figure 1. The reconstructed images and differential magnitude detection limits $\Delta \mathrm{m}$ of the six CVs (left to right, top to bottom): IX Vel, AE Aqr, U Gem, SS Cyg, V884 Her, and AH Her. There is no indication of any companion within 100 mas of any of the six stars that is within $\sim 4$ mag of the CV in either the blue $(466 \mathrm{~nm})$ or the red $(\mathrm{H} \alpha$ or $716 \mathrm{~nm})$ narrowband filters, respectively. Neither is any companion detected within $\sim 4-6$ mag (blue filter) nor 5-8 mag (red filter) out to 1.2 arcsec from any of the CVs. See the text for details. 


\section{ACKNOWLEDGEMENTS}

The data presented in this paper are based on observations obtained at the international Gemini Observatory, a program of NSF's NOIRLab, which is managed by the Association of Universities for Research in Astronomy (AURA) under a cooperative agreement with the National Science Foundation on behalf of the Gemini Observatory partnership: the National Science Foundation (United States), National Research Council (Canada), Agencia Nacional de Investigación y Desarrollo (Chile), Ministerio de Ciencia, Tecnología e Innovación (Argentina), Ministério da Ciéncia, Tecnologia, Inovações e Comunicações (Brazil), and Korea Astronomy and Space Science Institute (Republic of Korea). This work was enabled by observations made from the Gemini North telescope, located within the Maunakea Science Reserve and adjacent to the summit of Maunakea. We are grateful for the privilege of observing the Universe from a place that is unique in both its astronomical quality and its cultural significance. Observations in the paper made use of the high-resolution imaging instruments Zorro and 'Alopeke, which were funded by the NASA Exoplanet Exploration Program and built at the NASA Ames Research Center by Steve B. Howell, Nic Scott, Elliott P. Horch, and Emmett Quigley. Zorro was mounted on the Gemini South telescope, and 'Alopeke on the Gemini North telescope of the international Gemini Observatory. We thank the Canadian Gemini Time Allocation Committee for excellent feedback and support, and their allocation of telescope time. The observations were obtained under Gemini proposal GN2020A-Q-110. MMS thanks Nathan Leigh and Silvia Toonen for stimulating conversations about the likelihood and origins of triple companions to CVs. We acknowledge helpful suggestions from the referee which improved an earlier version of this paper.

\section{DATA AVAILABILITY STATEMENT}

The data underlying this article will be shared on reasonable request to the corresponding author. The raw speckle data are also available on the Gemini archive.

\section{REFERENCES}

Applegate J. H., 1992, ApJ, 385, 621

Beuermann K. et al., 2010, A\&A, 521, L60

Borges B. W., Baptista R., Papadimitriou C., Giannakis O., 2008, A\&A, 480, 481

Boyd D., 2012, J. Am. Assoc. Var. Star Obs., 40, 295

Bruch A., 2014, A\&A, 566, A101

Chavez C. E., Tovmassian G., Aguilar L. A., Zharikov S., Henden A., 2012, A\&A, 538, A122

Chavez C. E., Aviles A., Georgakarakos N., Ramos C., Aceves H., Tovmassian G., Zharikov S., 2020, Rev. Mex. Astron. Astrofis., 56, 19

Chen X., Maxted P. F. L., Li J., Han Z., 2017, MNRAS, 467, 1874

Dai Z., Qian S., 2009, Ap\&SS, 321, 91

De K. et al., 2021, ApJ, 912, 19

Drake A. J. et al., 2014, MNRAS, 441, 1186

Dubus G., Otulakowska-Hypka M., Lasota J.-P., 2018, A\&A, 617, A26

Fuentes-Morales I. et al., 2021, MNRAS, 501, 6083

Goliasch J., Nelson L., 2015, ApJ, 809, 80

Green R. F., Schmidt M., Liebert J., 1986, ApJS, 61, 305

Hamers A. S., Rantala A., Neunteufel P., Preece H., Vynatheya P., 2021, MNRAS, 502, 4479

Hardy A. et al., 2015, ApJ, 800, L24
Henry T. J. et al., 2018, AJ, 155, 265

Hill C., 2016, 19th Cambridge Workshop on Cool Stars, Stellar Systems, and the Sun (CS19). Uppsala, Sweden, p. 50

Hillman Y., Shara M. M., Prialnik D., Kovetz A., 2020, Nat. Astron., 4, 886

Horch E. P., Howell S. B., Everett M. E., Ciardi D. R., 2012, AJ, 144, 165

Howell S. B., Nelson L. A., Rappaport S., 2001, ApJ, 550, 897

Howell S. B., Everett M. E., Sherry W., Horch E., Ciardi D. R., 2011, AJ, 142,19

Knigge C., Baraffe I., Patterson J., 2011, ApJS, 194, 28

Kraft R. P., 1962, ApJ, 135, 408

Kraft R. P., 1964, ApJ, 139, 457

Kundra E., Hric L., 2011, Ap\&SS, 331, 121

Lagos F., Schreiber M. R., Parsons S. G., Gänsicke B. T., Godoy N., 2020, MNRAS, 499, L121

Law W. Y., Ritter H., 1983, A\&A, 123, 33

Leigh N. W. C., Toonen S., Portegies Zwart S. F., Perna R., 2020, MNRAS, 496, 1819

Liu W., Qian S.-B., Zhi Q.-J., Han Z.-T., Wang Q.-S., Dong A.-J., 2021, MNRAS, 505, 677

Livio M., Pringle J. E., 1994, ApJ, 427, 956

Mace G. N. et al., 2018, ApJ, 854, 145

Marsh T. R. et al., 2014, MNRAS, 437, 475

Martínez-Pais I. G., Martín-Hernández N. L., Casares J., Rodríguez-Gil P., 2000, ApJ, 538, 315

Meyer F., Meyer-Hofmeister E., 1979, A\&A, 78, 167

Mróz P. et al., 2015a, Acta Astron., 65, 313

Mróz P. et al., 2015b, ApJS, 219, 26

Osaki Y., 1974, PASJ, 26, 429

Paczynski B., Sienkiewicz R., 1981, ApJ, 248, L27

Pala A. F. et al., 2020, MNRAS, 494, 3799

Parsons S. G., Marsh T. R., Copperwheat C. M., Dhillon V. S., Littlefair S. P., Gänsicke B. T., Hickman R., 2010, MNRAS, 402, 2591

Patterson J. et al., 2018, PASP, 130, 064202

Pilarčík L., Wolf M., Dubovský P. A., Hornoch K., Kotková L., 2012, A\&A, 539, A153

Pilarčík L., Wolf M., Zasche P., Vraštil J., 2018, New Astron., 60, 1

Prialnik D., Shara M. M., Shaviv G., 1978, A\&A, 62, 339

Qian S. B., Han Z. T., Fernández Lajús E., Zhu L. Y., Li L. J., Liao W. P., Zhao E. G., 2015, ApJS, 221, 17

Schaefer B. E., 2020, MNRAS, 492, 3343

Schreiber M. R., Zorotovic M., Wijnen T. P. G., 2016, MNRAS, 455, L16

Scott N. J., Howell S. B., Horch E. P., Everett M. E., 2018, PASP, 130, 054502

Shara M. M., Livio M., Moffat A. F. J., Orio M., 1986, ApJ, 311, 163

Skopal A., Bode M. F., Lloyd H. M., Drechsel H., 1998, A\&A, 331, 224

Starrfield S., Truran J. W., Sparks W. M., Kutter G. S., 1972, ApJ, 176, 169

Stephenson C. B., Sanduleak N., 1977, ApJS, 33, 459

Thorstensen J. R., Peters C. S., Skinner J. N., 2010, PASP, 122, 1285

Tokovinin A., 2018, ApJS, 235, 6

Tokovinin A., Thomas S., Sterzik M., Udry S., 2006, A\&A, 450, 681

Verbunt F., Zwaan C., 1981, A\&A, 100, L7

Walker M. F., 1954, PASP, 66, 230

Warner B., 1988, Nature, 336, 129

Warner B., 1995, Cataclysmic Binary Stars, Cambridge Astrophysics Series. Cambridge Univ. Press, Cambridge, UK

Watson C. A., Steeghs D., Dhillon V. S., Shahbaz T., 2007a, Astron. Nachr., 328,813

Watson C. A., Steeghs D., Shahbaz T., Dhillon V. S., 2007b, MNRAS, 382, 1105

Zorotovic M., Schreiber M. R., 2013, A\&A, 549, A95

This paper has been typeset from a $\mathrm{T}_{\mathrm{E}} \mathrm{X} / \mathrm{L} \mathrm{T}_{\mathrm{E}} \mathrm{X}$ file prepared by the author. 